\title{
Interstitial lung disease and lung cancer: Decisions, precision, and tuna fish sandwiches
}

\author{
Victor A. Ferraris, MD, PhD
}

\author{
From the Division of Cardiothoracic Surgery, Department of Surgery, University of Kentucky, Lexington, Ky. \\ Disclosures: Author has nothing to disclose with regard to commercial support. \\ Received for publication April 8, 2017; accepted for publication April 12, 2017; available ahead of print May 26, \\ 2017. \\ Address for reprints: Victor A. Ferraris, MD, PhD, Tyler Gill Professor of Surgery, Division of Cardiothoracic \\ Surgery, Department of Surgery, University of Kentucky, A301 Kentucky Clinic, 740 S Limestone Ave, Lex- \\ ington, KY 40536-0284 (E-mail: ferraris@uky.edu). \\ J Thorac Cardiovasc Surg 2017;154:1097-9 \\ $0022-5223 / \$ 36.00$ \\ Copyright (C) 2017 by The American Association for Thoracic Surgery \\ http://dx.doi.org/10.1016/j.jtcvs.2017.04.029
}

Some pulmonologists devote their careers to the study of the various aspects of interstitial lung disease (ILD). Books have been written about this complex and heterogeneous disease process. ${ }^{1}$ For the most part, thoracic surgeons have not read these books. We are occasionally called on to provide specimens for diagnosis, but the epidemiologic study of ILD suggests that this disease process is not prevalent (somewhere around 70-80 autopsy specimens per 100,000 had pathologic findings of ILD). ${ }^{2}$ Nearly half of the autopsied patients with ILD had idiopathic pulmonary fibrosis, which represents the end stage of this disease. In most patients with ILD, a cause is not identified, a fact that I remind my pulmonary medicine colleagues about every time that they ask me to perform an operative biopsy on a ventilated, hypoxic, very sick patient for whom they would like to have a tissue diagnosis. ${ }^{3}$ For most cardiothoracic surgeons, an in-depth understanding of ILD is neither justified nor practical; however, some knowledge of the broad categories that can lead to ILD is valuable in talking with both patients and their referring physicians. I have noticed that surgeons like mnemonics. Figure 1 shows a mnemonic that I resurrect every time I am confronted with a patient with ILD, especially when asked to get a diagnosis of ILD in a high-risk surgical candidate. Around 50\% or more of patients with ILD who surface to the consciousness of thoracic surgeons have so-called idiopathic origins of ILD, without much chance for meaningful treatment or reversal. Nevertheless, among the established causes of ILD with potentially treatable and sometimes reversible causes are a number of drug therapies, infections, and occupational or allergic exposures. One reason that a mnemonic like that shown in Figure 1 may be helpful to "outcomefocused" thoracic surgeons is that it sorts out potentially treatable causes of ILD and helps support the value of operations on certain patients with ILD who face a high operative risk.

The possibility that ILD and lung cancer are associated has been recognized for more than 50 years, but it remains unclear whether ILD precedes lung cancer or vice versa.

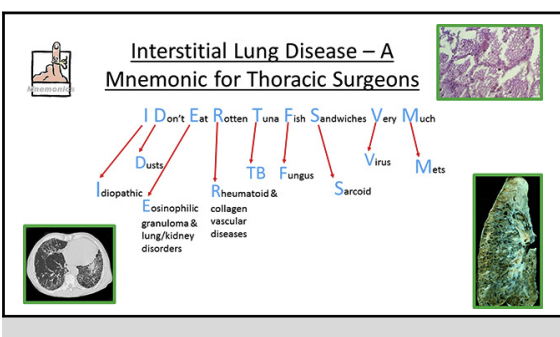

Mnemonic for interstitial lung disease.

Central Message

Lung cancer can coexist with interstitial lung disease and often benefits from sublobar resections, but careful diagnosis of interstitial lung disease can guide lung cancer resections.

See Article page 1089.

A systematic review of the association between lung cancer and ILD suggested that certain patients with well-defined ILD have widely varying levels of associated lung cancers. ${ }^{4}$ Table 1 summarizes the findings of this systematic review. Further, some treatments for lung cancer, including chemotherapy and radiation, may induce ILD. ${ }^{5}$ Perhaps the most intriguing association with ILD is cigarette smoking. There are at least 4 types of ILD with strong links to smoking. These include desquamative interstitial pneumonia, respiratory bronchiolitis-associated interstitial lung disease, pulmonary Langerhans cell histiocytosis, and idiopathic pulmonary fibrosis. Evidence suggests that most cases of desquamative interstitial pneumonia, respiratory bronchiolitis-associated interstitial lung disease, and pulmonary Langerhans' cell histiocytosis are caused by cigarette smoking in susceptible individuals. ${ }^{6,7}$ Smoking cessation should be a main component in the initial therapeutic approach to smokers with these interstitial lung diseases. In addition, smoking appears to be a risk factor for the development of idiopathic pulmonary fibrosis. Given the known risks of lung cancer in smokers and the associated smoking-related ILD, it is not a stretch to suggest that lung cancer might have a fairly strong association with ILD.

It is thus not surprising that the study by Okada and coauthors $^{8}$ appearing in this issue of the Journal documents a population of patients with ILD undergoing operations for early-stage lung cancers. The article describes their 


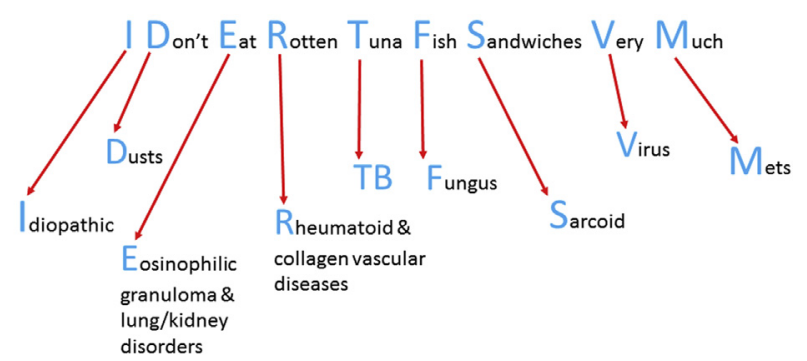

FIGURE 1. Interstitial lung disease: a mnemonic for thoracic surgeons. $T B$, Tuberculosis; Mets, metastatic cancers.

experience with resection of suspected clinical stage I lung cancer in 107 patients with ILD. ${ }^{8}$ They simplified characteristics of patients with ILD by dividing them into 3 categories according to imaging characteristics from computed tomographic (CT) scans: usual interstitial pneumonitis (UIP), possible UIP (p-UIP), and not consistent with UIP (non-UIP). They found that patients with UIP or p-UIP had significantly decreased survival relative to nonUIP patients after resection for early-stage lung cancer. If one had to guess, it is likely that patients with UIP and pUIP have the idiopathic form of ILD, possibly complicated by interstitial fibrosis. These 2 groups may represent patients with more advanced stages of ILD, and one might suspect decreased survival in these 2 groups, not necessarily related to early stage lung cancer but more likely related to advanced underlying ILD complicated by coexisting early-stage lung cancer. Figure 2 in the article of Okada and coauthors ${ }^{8}$ supports this notion. There is very likely an incremental mortality risk in patients with lung cancer who have the idiopathic form of ILD. There is an obvious lack of precision in the diagnosis of ILD among the patients undergoing lung cancer resections. The categorization of patients with ILD is imprecise, but it is useful and provocative.

The main advantage of sublobar resection is preservation of lung function. The question is, to what extent does residual lung function determine overall survival in patients with lung cancer complicated by ILD? From the results of Okada and coauthors, ${ }^{8}$ preoperative lung function does not make a significant contribution to overall survival, but there is an intriguing if nonsignificant trend toward increased survival with sublobar resection that is clouded by small sample

TABLE 1. Incidences of lung cancer in patients with interstitial lung disease of various etiologies ${ }^{4}$

\begin{tabular}{|c|c|c|}
\hline $\begin{array}{c}\text { Type of interstitial lung } \\
\text { disease }\end{array}$ & $\begin{array}{l}\text { Incidence range } \\
\text { of lung cancer }\end{array}$ & $\begin{array}{c}\text { No. of patients/ } \\
\text { No. of studies }\end{array}$ \\
\hline Idiopathic pulmonary fibrosis & $2 \%-63 \%$ & $1444 / 3$ \\
\hline Sarcoidosis & $1 \%-37 \%$ & $7730 / 4$ \\
\hline Scleroderma & $3 \%-12 \%$ & $1462 / 5$ \\
\hline Dermatomyositis/Polymyositis & $20 \%-39 \%$ & $3367 / 3$ \\
\hline Rheumatoid arthritis & $0.7 \%-16 \%$ & $101,931 / 5$ \\
\hline Systemic lupus erythematosus & $0.3 \%-0.6 \%$ & $19,663 / 3$ \\
\hline
\end{tabular}

size. The exact amount of ILD left in patients after resection is uncertain from this study and may contribute to overall survival. Okada and coauthors ${ }^{8}$ conclude, perhaps inappropriately but with justifiable speculation, that patients with either UIP or p-UIP may benefit from sublobar resection despite the finding of a nonsignificant difference $(P=.07)$ between patients undergoing lobectomy versus sublobar resection.

Okada and coauthors ${ }^{8}$ divided ILD into 3 categories according to CT scan findings. This is an obvious oversimplification. The differential diagnosis of ILD is enormous, and Figure 1 is a little more rigorous than the definition of Okada and coauthors ${ }^{8}$ but is still a simplified version. The diagnostic categories of ILD are enormous. The most common diagnostic category is UIP, but many other possibilities exist and cannot always be separated by $\mathrm{CT}$ findings alone. Things such as hypersensitivity pneumonitis, collagenvascular disease, infections (fungal and tubercular), dusts, sarcoid, and even metastatic cancer can give a picture of ILD on CT scan. Some of these other diagnoses might well affect survival. I wonder whether more specific diagnoses of ILD were made after lung cancer resections in the patients of Okada and coauthors. ${ }^{8}$ Pathologists tell me that the routine studies done on resected lung cancer specimens are much less intense than histopathologic studies performed in lung specimens from patients with suspected ILD. In patients with ILD undergoing resections for lung cancer, it is important to subject the pathologic specimens to a very rigorous histopathologic assessment that helps to define categories of ILD in resected specimens.

It might be interesting to assess ILD on postoperative CT scans to determine the extent of ILD remaining in patients after cancer resection. This would involve quantitative assessment of abnormal lung from chest imaging, something that is possible but tedious. ${ }^{9}$ The clinical significance of this postoperative assessment might give some information that clinicians could use to guide therapy and provide prognosis. If overall survival correlated with the amount of residual ILD after resection, this might give a better idea of the importance of ILD coexisting in patients with lung cancer. It also might guide surgeons with respect to whether future sublobar resections should be done selectively in patients to preserve lung not involved with ILD. Really, the important thing about lung cancer resection in patients with ILD is how much relatively normal lung is being removed, and Okada and coauthors ${ }^{8}$ have not assessed the impact of resection on residual lung function. Importantly, they have not described their surgical approach to patients with ILD. There is no indication that they changed their operative approach according to whether or not the lung cancer being resected was in an area of ILD or in an area of relatively normal lung function. There are some unanswered questions that surface from this intriguing study, and I hope that this study stimulates further work that 
addresses the impact of lung cancer resection on ILD progression. As Okada and coauthors ${ }^{8}$ point out, the "choice of surgical approach is not determined by postoperative factors." Unfortunately, it is not possible to be precise about the diagnosis of ILD on the basis of preoperative imaging, but postoperative histopathologic assessments of ILD may help with patient management and help future patients with ILD and lung cancer.

\section{References}

1. Schwarz MI, King TE Jr. Interstitial lung disease. 5th ed. Lewiston (NY): B.C. Decker; 2011.

2. Coultas DB, Zumwalt RE, Black WC, Sobonya RE. The epidemiology of interstitial lung diseases. Am J Respir Crit Care Med. 1994;150:967-72.
3. Raghu G, Nyberg F, Morgan G. The epidemiology of interstitial lung disease and its association with lung cancer. Br J Cancer. 2004;91(Suppl 2):S3-10.

4. Archontogeorgis K, Steiropoulos P, Tzouvelekis A, Nena E, Bouros D. Lung cancer and interstitial lung diseases: a systematic review. Pulm Med. 2012;2012. 315918.

5. Camus $\mathrm{P}$, Kudoh S, Ebina M. Interstitial lung disease associated with drug therapy. Br J Cancer. 2004;91(Suppl 2):S18-23.

6. Ryu JH, Colby TV, Hartman TE, Vassallo R. Smoking-related interstitial lung diseases: a concise review. Eur Respir J. 2001;17:122-32.

7. Vassallo R. Diffuse lung diseases in cigarette smokers. Semin Respir Crit Care Med. 2012;33:533-42.

8. Tsutani Y, Mimura T, Kai Y, Ito M, Misumi K, Miyata Y, Okada M. Outcomes after lobar versus sublobar resection for clinical stage I non-small cell lung cancer in patients with interstitial lung disease. J Thorac Cardiovasc Surg. 2017;154: 1089-96.e1.

9. Uppaluri R, Mitsa T, Sonka M, Hoffman EA, McLennan G. Quantification of pulmonary emphysema from lung computed tomography images. Am J Respir Crit Care Med. 1997;156:248-54. 